\title{
Reviews of Accelerator Science and Technology
}

\author{
Volume 3
}


This page intentionally left blank 


\section{Reviews of}

Accelerator Science

and Technology

Volume 3

Accelerators as Photon Sources

\section{Editors}

\section{Alexander W. Chao}

Weiren Chou

SLAC National Accelerator Laboratory, USA

Fermi National Accelerator Laboratory, USA 


\section{Published by}

World Scientific Publishing Co. Pte. Ltd.

5 Toh Tuck Link, Singapore 596224

USA office: 27 Warren Street, Suite 401-402, Hackensack, NJ 07601

UK office: 57 Shelton Street, Covent Garden, London WC2H 9HE

\section{British Library Cataloguing-in-Publication Data}

A catalogue record for this book is available from the British Library.

\section{REVIEWS OF ACCELERATOR SCIENCE AND TECHNOLOGY \\ Volume 3: Accelerators as Photon Sources}

Copyright (c) 2010 by World Scientific Publishing Co. Pte. Ltd.

All rights reserved. This book, or parts thereof, may not be reproduced in any form or by any means, electronic or mechanical, including photocopying, recording or any information storage and retrieval system now known or to be invented, without written permission from the Publisher.

For photocopying of material in this volume, please pay a copying fee through the Copyright Clearance Center, Inc., 222 Rosewood Drive, Danvers, MA 01923, USA. In this case permission to photocopy is not required from the publisher.

ISBN-13 978-981-4340-38-0

Printed in Singapore by Mainland Press. 


\section{Contents}

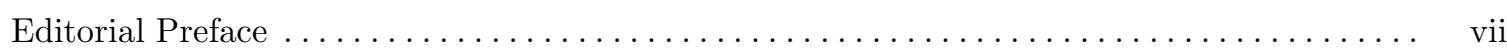

Invention of the Free Electron Laser

John M. J. Madey ......................................... 1

Photon Science at Accelerator-Based Light Sources

Jochen R. Schneider ...................................... 13

Electromagnetic Radiation in Accelerator Physics

G. Stupakov.................................................. 39

Storage Ring Light Sources

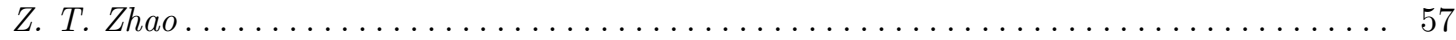

Low-gain Free Electron Lasers

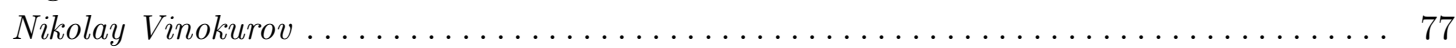

Soft and Hard X-ray SASE Free Electron Lasers

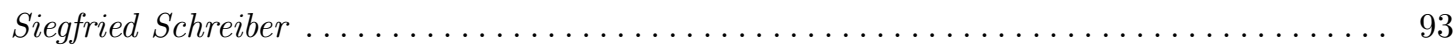

Energy Recovery Linacs for Light Sources

Ryoichi Hajima............................................... 121

Compton Sources of Electromagnetic Radiation

Geoffrey A. Krafft and Gerd Priebe ...................................... 147

Accelerator-Based Sources of Infrared and Terahertz Radiation

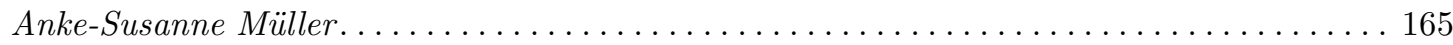

The Next Generation of X-ray Sources

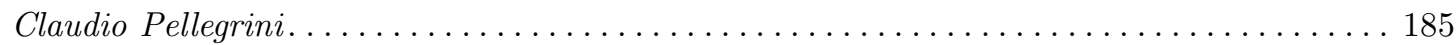

Undulators and Other Insertion Devices

Eugene Levichev and Nikolay Vinokurov .............................. 203

High Performance Electron Injectors

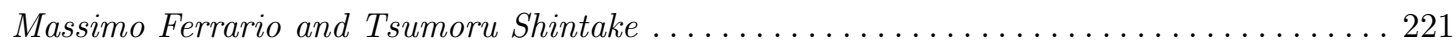

Electron-Beam-Based Sources of Ultrashort X-ray Pulses

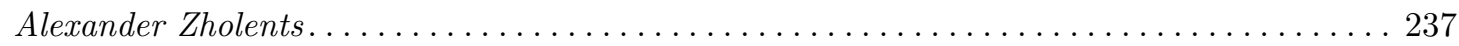

The Large Hadron Collider from Conception to Commissioning: A Personal Recollection

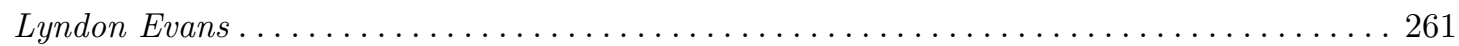

G. I. Budker: Brilliant Physicist, Great Scientific Leader

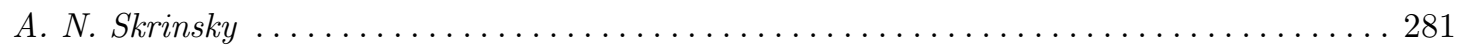


This page intentionally left blank 


\section{Editorial Preface}

Since its inception in 2008, Reviews of Accelerator Science and Technology (RAST) has strived to serve as a journal for the worldwide accelerator community. The theme of each volume of $R A S T$ has been chosen with wide community input and advice. The theme of Volume 1 (2008) was an overview across the entire accelerator field. The theme of Volume 2 (2009) was "Medical Applications of Accelerators," because of the enormous importance to human health of this topic and its deep impact on society. The theme of Volume 3 is "Accelerators as Photon Sources."

Photon emission by electrons in an accelerator was first observed in 1946 in a synchrotron at the General Electric Research Laboratory in Schenectady, New York State, USA (hence the term "synchrotron radiation"). This discovery opened a new era of accelerator-based photon sources. Over the last half-century, we have witnessed tremendous progress in this field. This dramatic evolution has seen four generations of accelerators as photon sources. The first generation employed the electron storage rings built primarily for high energy physics experiments, and the synchrotron radiation from the bending magnets was used parasitically. The second generation was comprised rings dedicated to synchrotron radiation applications, with the radiation again from the bending magnets. The third generation, currently the workhorse of these photon sources, consists of dedicated advanced storage rings that employ not only bending magnets but also insertion devices (wigglers and undulators) as the source of the radiation. The fourth generation, which is just entering operation, comprises photon sources based on the free electron laser (FEL), an invention made in the early 1970s.

Each generation produced increases in brightness and time resolution that had been unimaginable just a few years earlier. In particular, the progression from the third to the fourth generation is a true revolution; the peak brilliance of coherent soft and hard X-rays has increased by 7-10 orders of magnitude, and the image resolution has reached the angstrom $\left(1 \AA=10^{-10} \mathrm{~m}\right)$ and femtosecond $\left(1 \mathrm{fs}=10^{-15} \mathrm{~s}\right)$ scales. These impressive capabilities have fostered fundamental scientific advances and led to an explosion of numerous possibilities in many important research areas, including materials science, chemistry, molecular biology, and the life sciences. Even more remarkably, this field of photon source invention and development shows no signs of slowing down. Studies have already been started on the next generation of X-ray sources, which would have a time resolution in the attosecond $\left(1 \mathrm{as}=10^{-18} \mathrm{~s}\right)$ regime, comparable to the time of electron motion inside atoms. It can be fully expected that these photon sources will stand out among the most powerful future science research tools. The physics community — indeed, the entire science community — will hear of many pioneering and groundbreaking research results involving these sources in the coming years. It is timely and appropriate that RAST devotes Volume 3 to this topic.

This volume contains 15 articles, all written by leading scientists in their respective fields. The first 13 are on "Accelerators as Photon Sources." The first article is by John Madey, inventor of the free electron laser. He also coined the name "free electron laser," and he carried out the landmark FEL experiments. Following Madey's article is a review of photon science (Schneider) and a review of radiation electrodynamics (Stupakov). Then there are three articles describing and discussing third and fourth generation photon sources (Zhao, Vinokurov, and Schreiber).

The next four articles are devoted to ideas, concepts, and research and development of future sources (Hajima on energy recovery linacs, Krafft and Priebe on Compton sources, Müller on terahertz sources, and Pellegrini on advanced source concepts). Three articles then review the technologies that will be critical in future photon source development (Levichev and Vinokurov on insertion devices, Ferrario and Shintake on high performance electron injectors, and Zholents on ultrashort X-ray pulses). 
The Large Hadron Collider (LHC) at CERN is the largest accelerator in the world, with a circumference of $27 \mathrm{~km}$. It has attracted enormous attention worldwide since it began operation this year, because of its mega size and unprecedented potential for exploration of the fundamental mysteries of our universe, the nature of matter and antimatter, and dark energy — to name but a few. We invited Lyndon Evans, the LHC project leader from 1993 to 2008, to write a review of the LHC for this volume. Also in the volume is an article by Sasha Skrinsky dedicated to Gersh Budker, our choice for "Person of the Issue." Budker was one of the true giants of Russian science and he perceived science as a truly international enterprise, as will be told in the last article of this volume.

Since its inception in 2008, this journal has garnered strong support from the accelerator community. We have received invaluable advice and excellent suggestions from the Editorial Board, and from our colleagues and friends. We have enjoyed good reviews and experienced great cooperation from all the authors and referees. With RAST entering its third year, although it is still young, we believe that the foundation of this journal has been laid, and it has made good progress toward meeting our goal of its serving as an annual journal for the international accelerator community. We are grateful for the valuable support from the community.

Alexander W. Chao
SLAC National Accelerator Laboratory, USA
achao@slac.stanford.edu
Weiren Chou
Fermi National Accelerator Laboratory, USA
chou@fnal.gov
Editors

